\title{
sciendo
}

Transport and Telecommunication, 2021, volume 22, no. 1, 29-38

Transport and Telecommunication Institute, Lomonosova 1, Riga, LV-1019, Latvia DOI 10.2478/ttj-2021-0003

\section{A VEHICULAR QUEUE LENGTH MEASUREMENT SYSTEM IN REAL-TIME BASED ON SSD NETWORK}

\section{Wahban Al Okaishi', Abdelmoghit Zaarane', Ibtissam Slimani, Issam Atouf ${ }^{4}$, Mohamed Benrabh ${ }^{5}$}

\author{
1,2,3,4,5 LTI Lab. Faculty of Sciences Ben M'sik, \\ Hassan II-Casablanca University \\ Casablanca, Morocco \\ 'wahbanyahya@gmail.com
}

\begin{abstract}
Vehicular queue length measurement is an important parameter to detect the traffic congestion, which is resulted from several issues such as traffic lights, accidents, and poor roads infrastructures. In this paper, a system in real-time is proposed to detect and measure the vehicular queue length at intersections. The proposed system consists of two main steps: the first step is the detection of queue by using frames differencing method to detect the motion in the target areas. If there is no a motion, then the second step is implemented to detect the vehicles in these areas by using Single Shot Multibox Detector (SSD) algorithm. If there are vehicles, that means the queue exists and the measurement process begins. Some modifications are applied on SSD algorithm to fit with in our system and to improve the accuracy of the vehicle detection process. The system is applied on videos obtained by stationary cameras. The experiments demonstrate that this system is able to accurately detect and measure the vehicular queue length.
\end{abstract}

Keywords: Image processing, Vehicle detection, Motion detection, queue length measurement, Single Shot Multiboxs Detector (SSD)

\section{Introduction}

Several systems have been proposed for analysis of traffic parameters. The old ones (Burnos et al., 2007) (Sun et al., 1998) utilized a lot of detectors (e.g. loop, radar, infrared ultrasonic, and microwave) to collect and detect traffic parameters. However, these detectors have numerous drawbacks (e.g. their installation requires pavements cut, and the detection zone is small), that restrict their use.

In recent years, vision-based systems have been widely utilized to collect and control traffic parameters due to their advantages compared with old detectors. Therefore, in this paper, we propose a vision-based system to implement the measurement of vehicular queue length. The vehicular queue length is an important traffic parameter, which is used for quantitative analysis of traffic scenes. Various systems were implemented to measure this parameter. However, these systems are based on traditional image processing algorithms for detecting vehicles such as edge detection (Slimani et al., 2018) (Al Okaishi et al., 2019a) (Fathy and Siyal, 1995), and corner detection (Albiol et al., 2011a) (Chintalacheruvu and Muthukumar, 2012) (Albiol et al., $2011 \mathrm{~b}$ ). Due to the utilization of these algorithms, the performance of these systems can be affected by several issues such as weather conditions, shadows, the marks on the roads, and etc. Most of queue length measurement systems are based on two steps: the first step is to detect the vehicular queue by using the motion detection algorithm. If there is no a motion, then the second step is performed by using vehicle detection. If there are vehicles, then there is a queue and the measurement process is implemented.

In this paper, a system in real-time is proposed to detect and measure the vehicular queue length at intersections. The proposed system consists of two main steps: the first step is the detection of queue by using frames differencing method to detect the motion in the target areas as shown in Figure 1.

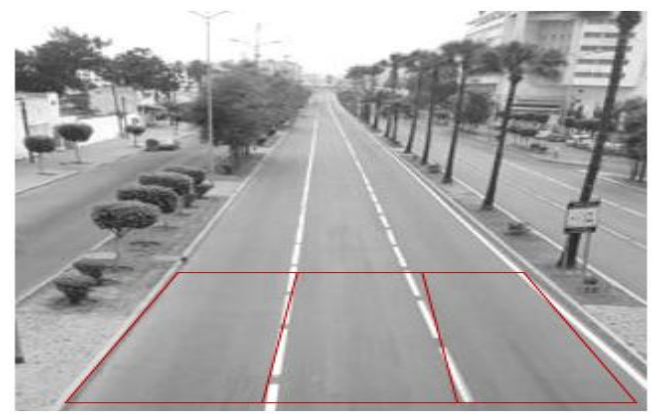

Figure 1. The First Detection Areas in each lane 
If there is no a motion, then the second step is implemented to detect the vehicles in these areas by using SSD algorithm. If there are vehicles, that means the queue exists and the measurement process begins. Some modifications are applied on SSD algorithm to fit with in our system and to improve the accuracy of the vehicle detection process. The system is applied on videos obtained by stationary cameras.

The rest of this paper is organized as follows: In Section 2, we present the related works. Section 3 we describe the proposed system, which includes three subsections: motion detection, vehicle detection, and the flowchart of our proposed system. Section 4 describes the experimental results and their evaluations. Finally, the conclusion is given in Section 5.

\section{Related works}

In this section, we highlight the previous queue length measurement systems. Several systems were developed for implementing the measurement of vehicular queue length. In (Rourke and Bell, 1991), the authors proposed a system to detect the vehicular queue. However, their system cannot measure the queue length. Fathy and Siyal (1995) developed a system to detect and measure the vehicular queue length. Their system is based on dividing the road scene into subprofiles, which has a size determined by camera and geometry parameters. The system consists of two principle steps: motion detection and vehicle presence detection. Firstly, the motion detection is applied on the first subprofiles to insure that there are no moving vehicles. If there is no motion, then the vehicle presence detection algorithm is applied by using the edge detection. If the vehicles are detected, then there is a vehicular queue and the system begins to measure the queue length. In another work (Siyal and Fathy, 1999), they utilized the neural network to train the output of vehicle presence to estimate the queue length.

The system in (Iwasaki, 1997), was implemented to improve the timing of traffic light. The traffic scene in this work is divided each lane into blocks and the vehicle detection was performed by using Sobel edge detection operator. A system in (Zanin et al., 2003), was proposed to measure the vehicular queue length. The authors utilized the phase of Gabor transformed and the frame differencing method to detect the queue. With regard to the measurement of the queue, they compute the horizontal edges to detect vehicles by using five levels directional Robinson convolutional kernel. The authors in (Albiol et al., 2011a), used another feature for detecting vehicles which is corner feature. Their system is based on using Harris corner detector for detecting vehicles to estimate the queue length at the individual lanes of the road. The proposed system in (Shirazi and Morris, 2015), detects and estimates the queue length by tracking the vehicles. They determined an area on the road called the motion area, which is in a location where the vehicles still move, and in the same time it must to be close to the camera. The background subtraction method was used to detect moving vehicles in this area, then the detected vehicles are tracked until they stop. The estimation of the queue length is determined by counting the vehicles that cross the motion area. The drawback of this system is that the motion detection area must be close to the camera in order to easily detect the vehicles but this leads to limit the queue length by the location of the motion area. So the system has no ability to measure the queue length behind the motion detection area.

As previously mentioned, all previous systems utilized the traditional image processing methods such as edge detection, corner detection to detect stopped vehicles, and background subtraction to detect moving vehicles. These methods are affected by numerous factors such as weather conditions, shadows, the marks on the roads, the speed of vehicles, and etc. In this paper, a method based on deep learning technique is proposed for vehicle detection. The SSD network is used to robustly detect the vehicles in the proposed system, which is not affected by the above-mentioned factors.

\section{The proposed system}

In this section, the proposed system is explained in details. The proposed system is based on two principle methods: motion detection method, and vehicle detection method.

\subsection{Motion Detection}

The first step to measure the vehicular queue length is to detect if there is a queue or not. In this paper, the motion detection is used to implement this task. Firstly, the motion detection algorithm is applied on the detection motion areas in each lane of the road as shown in Figure 1.

To increase the performance of the proposed system, in addition to respecting our needs. We use a simple and effective method called frame differencing. It detects the motion in the target area by comparing each pixel in the target area in the successive frames. If the difference is large enough, then there is a motion 
in this area. There are situations when some differences are resulted by noise, or camera vibration. However, these differences do not affect the performance of this method because they are very low compared with the differences resulted by the vehicle motion. Figures 2, 3 show the histogram of the difference when there is a motion and no a motion respectively.

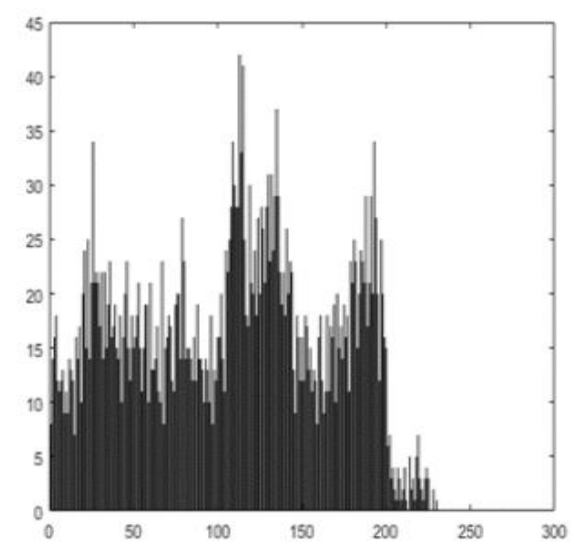

Figure 2. The histogram of the difference when there is a motion

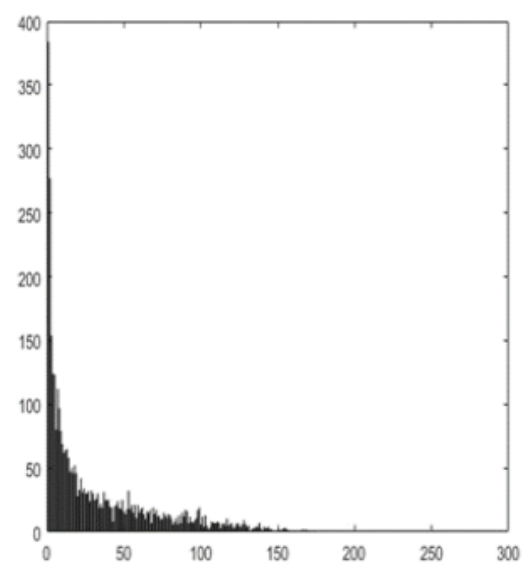

Figure 3. The histogram when there is no motion

The mean of the difference of the target area pixels $M_{d}$ is calculated by equation (2):

$\mathrm{D}=\left|\mathrm{I}_{\mathrm{t}-1}(\mathrm{x}, \mathrm{y})-\mathrm{I}_{\mathrm{t}}(\mathrm{x}, \mathrm{y})\right|$,

$\mathrm{M}_{\mathrm{d}}=\sum_{\mathrm{i}=1}^{\mathrm{N}} \frac{\mathrm{D}(\mathrm{x}, \mathrm{y})}{\mathrm{N}}$,

where $I_{t-1}$ is the pixel value in the previous frame, $I_{t}$ is the pixel value in the current frame, and $N$ is the number of pixels in the target area. If the mean is larger than a specific threshold, then there is a motion. After several experiments in different weather conditions and various situations, we found that the interval [18-22] is the best range to choose the threshold. In this work, we choose 20 as a predefined threshold to determine if there is a motion or not in the target areas (Al Okaishi et al., 2019).

\subsection{Vehicle Detection}

Over the last few decades, various methods have been developed for detecting vehicles. These methods can be divided into two categories: traditional image processing methods, and deep learning-based methods. In first category, the background subtraction (Sen-Ching and Kamath, 2004) (Al Okaishi et al., 2019a) is widely used to detect moving vehicles, while the texture detection (e.g. edge detection, corner detection, and etc.) are utilized to detect stopped vehicles (Al Okaishi et al., 2019b) (Albiol et al., 2011a). Another type of traditional methods is the low-level image features extractors such as SIFT (Lowe, 2004), HOG (Dalal and Triggs, 2005), and LBP (Tan and Triggs, 2007), which are utilized to extract vehicles' features from images.

Recent years have witnessed tremendous development in object detection by using deep convolutional neural networks (CNNs) because the CNN structures help to understand the images. In addition, they have higher accuracy and outperform the traditional methods. Therefore, they are widely utilized in pattern recognition and image processing. These networks can be region proposal-based networks (e.g. Region-based CCN (R-CNN), Fast R-CNN, Faster R-CNN) or regression-based networks (e.g. YOLO (You Only Look One), SSD).

CNN architecture includes one or more convolutional layers with a pooling layers (average, or max), and are followed by one or more fully connected layers. CNNs have numerous architectures available nowadays (e.g. PatroNet (Nogueira et al., 2015), AlexNet (Krizhevsky et al., 2012), VGG16 (Simonyan and Zisserman, 2014), and etc.). These architectures well classify the objects, however, they have no ability to localize them in images.

Therefore, several searches have been proposed to localize the objects in images based on CNNs. R-CNN (Girshick et al., 2015a) is one of the first algorithms, which utilized CNN in object detection systems and had a much higher performance than other popular methods. The idea of this method is the use of the selective search algorithm to generate regions in an image that could belong to a particular object which are called region proposals (2000 category independent region proposals are generated for each individual image). $\mathrm{CNN}$ is implemented to extract a 4096 dimensional feature vector from each of 
the 2000 region proposal. After training CNN, the feature vector is obtained from the penultimate layer of CNN. This vector is the input of the next stage (which is SVM (Support Vector Machine)). This method suffers from several drawbacks such as the slow of object detection, and the training process is multistage pipeline, in addition it consumes a huge memory. The authors of (Girshick et al., 2015b) proposed an algorithm called Fast R-CNN to address these drawbacks by using Special Pyramid Pooling networks (SPPnets). The feature vector, which is used to classify the objects in this method, is extracted by using SSPnets. The training process as also multistage pipeline and also consumes a huge memory just like $\mathrm{R}-\mathrm{CNN}$, however, it is faster and has higher detection accuracy than R-CNN.

Another algorithm was developed for object detection based on CNN called Faster R-CNN (Ren et al., 2015), which includes two modules: Region Proposal Network (RPN), which generates region proposals, and a Fast R-CNN module, which uses these proposal to detect the objects. Faster R-CNN generates the entire system of object detection in a single, unified network through sharing the common set of convolution layers for both modules. This method reduces the amount of time and memory better than previous methods.

New networks have been proposed to address the bottlenecks of previous methods. The most popular networks are YOLO (Redmon et al., 2016), and SSD network (Liu et al., 2016). YOLO detects the objects in images by dividing every image into a grid of D x D and every grid predicts $\mathrm{N}$ bounding boxes and categories. DxDxN bounding boxes are proposed in each image. However, SSD has performances better than YOLO network. Because SSD has higher accuracy than YOLO.

SSD utilized VGG16 as a base network to generate the first feature map from layer 23, which detects the small objects and has a size of 38x38 and depth of 512. The second feature map detects slightly larger objects because it covers bigger receptive fields, and this feature map has a size of 19x19 and depth of 1024. Down to the last layer, which produces a feature map that can be ideally used to detect big objects. Every feature map is connected by two layers: the first one is called category prediction layer that is used to predict the class of object, and the other is called bounding box prediction layer that is used to predict the bounding box. SSD suffers from missed detection for small objects.

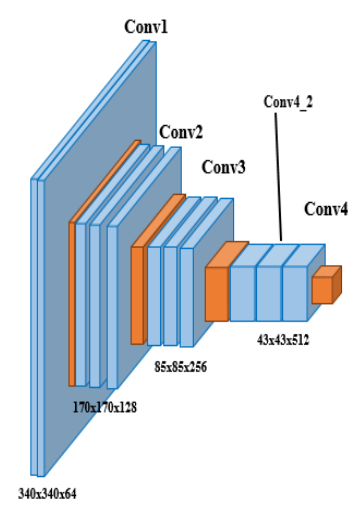

Figure 4. The base network in our model

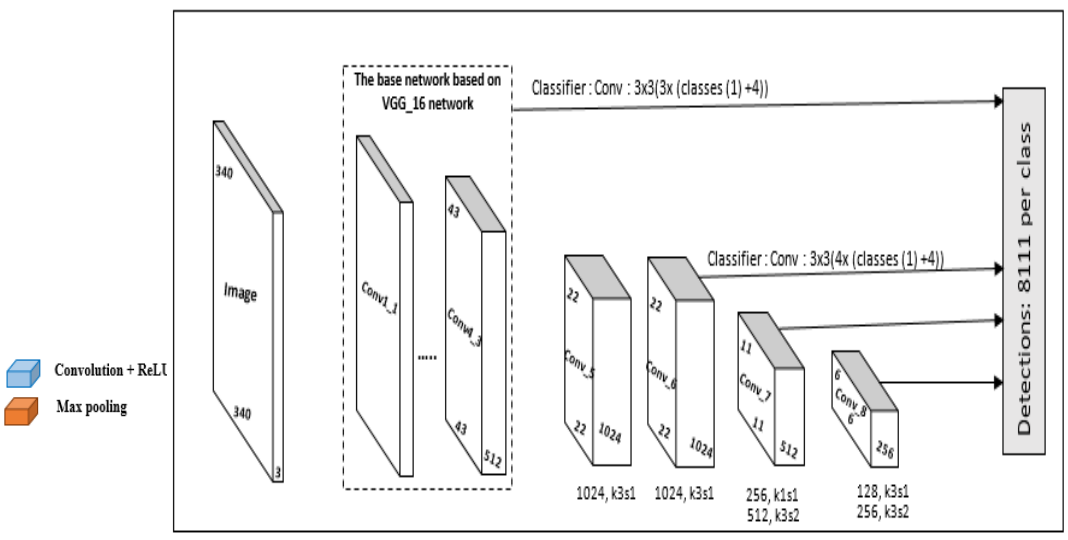

Figure 5. The proposed model

In this paper, some modifications are applied on SSD algorithm to fit with in our system and to improve the accuracy of the vehicle detection process. SSD network is a general detector, and this means it is implemented to detect any object with different scales and aspect ratios in images. In our situation, we need to only detect the vehicles. Therefore, many modifications are applied on SSD network to fit in with our system, which lead to improve the accuracy of the vehicle detection process and the entire system. As the aspect ratio of different vehicles (Huang et al., 2012) is in a range [1.3-2]. Therefore, we set the aspect ratio to 1.5 , and 2 (instead of aspect ratios of original SSD $0.3,0.5,1,2$, and 3 ). As our system is applied on images that contains of a queue of vehicles, the vehicles sizes in these images are small compared with the image size. Therefore, the last two feature maps of SSD are removed from the architecture. To address the bottleneck of SSD, we modify the architecture of the base network and other layers to better detection for small objects as follows: The training images are the input of the base network. It consists of four blocks, each block is considered as the combination of a convolutional layer (the resolution of the block layers is the same by using the padding), followed by a semilinear activation function layer (ReLU - Rectified Linear Unit). The last layer of the block is followed by a maximum pooling layer. The first block has two convolutional layers, each of them has 64 filters with size of 3x3 and stride of 1 . The output of the first block is the input of the second block. The configuration of the rest blocks is similar to the first block. Figure 4. shows the architecture of the base network. 
Due to SSD network suffers from missed detection for small objects, we focus on how to improve the detection of the small objects. The base network in our model produces a feature map with size of $43 \times 43$ and depth of 512 as shown in Figure 4, which covers smaller receptive fields, and better detects the small objects. The base network is followed by four extra convolutional layers, three of them produces feature maps with smaller size than the previous layer to cover bigger receptive field for detecting bigger objects as shown in Figure 5.

Four default bounding boxes with different aspect ratios and scales are proposed for each cell in all the feature maps, except the first feature map which has three default bounding boxes for each cell. For an mxn feature map, there are mxnx4 or mxnx3 bounding boxes and each bounding box has (c +4$)$ outputs, where $\mathrm{c}$ is the number of classes (in this paper, $\mathrm{c}=1$ ). For each anchor box (default bounding box), the anchor box is considered as a positive box, if its IoU (Intersection over Union) is higher than a predefined threshold, and it has the largest IoU with the ground-truth bounding box. Otherwise, it is considered as a negative box.

\subsection{The flowchart of the proposed system}

In this paper, a system in real-time to detect and estimate the vehicular queue length is proposed. The flowchart of the proposed system is shown in Figure 6.

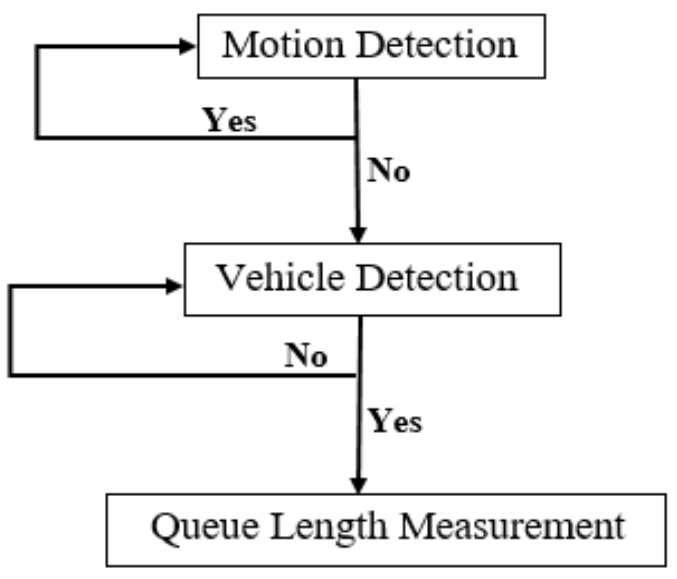

Figure 6. The flowchart of the proposed system

The proposed system involves the following steps:

- The first step is to detect the queue at the head of the queue (in the target areas as shown in Figure 1) by using the motion detection method. The motion detection method is continuously applied on the head of the queue.

- If there is a motion, then there is no queue. Therefore, the motion detection method is continuously applied on the head of the queue until detecting no motion.

- If there is no motion, then the vehicle detection method is applied to ensure that there are vehicles.

- If there are vehicles in these areas, then the measurement of the vehicular queue length begins, by adding the height of the bounding boxes of the detected vehicles.

- After detecting vehicles at the head of the queue, the vehicle detection method is applied on the area just behind the previous area. If there are vehicles, then the queue length is increased by the height of the bounding boxes of the detected vehicles. If there are no vehicles, then the vehicle detection method waits for 3 seconds to be again applied on the same area.

- Then the vehicle detection method is applied on behind the previous area and repeat the process.

- If the motion is detected in the head of the queue, this means the vehicles started to move, thus the queue length will fall to zero immediately. During the measurement of the queue length, when the motion is detected in the head of the queue, the queue will be destroyed even if the vehicle in the head of the queue moves slowly and then stops. This leads the system to be unstable through the time. To cope with this problem, the time of transition between the no motion state and motion state $\mathrm{T}_{\mathrm{nm}}$ must be sufficient to ensure the continuous movement of vehicles. If there is motion for time less than $\mathrm{T}_{\mathrm{nm}}$, it is ignored (Al Okaishi et al., 2019a). 


\section{Experimental results}

This section is divided into two parts: The first part is for evaluating the proposed vehicle detection method, while the other one is for evaluating the entire system (The measurement of vehicular queue length).

\subsection{Evaluation of the proposed vehicle detection method}

In this work, we utilize SSD network with improvements to detect the different vehicles. The proposed method is trained by using MIO-TCD dataset (MIO-TCD dataset, 28). This dataset contains of two parts: One for classification, and one for localization. It includes images for 11 classes of vehicles. We utilized 12000 images for training the proposed network, and 8000 for testing. All used images are relabelled to one class (vehicle).

We conducted a comparative study to evaluate our vehicle detection method. We compared our method with the most three famous vehicle detection methods (Faster R-CNN, YOLO2, and SSD512). After training the different methods by using the same dataset, we apply them on two different videos. These videos were taken from stationary traffic cameras of Casablanca city. Figure 7. shows the result of vehicle detection for the different methods.
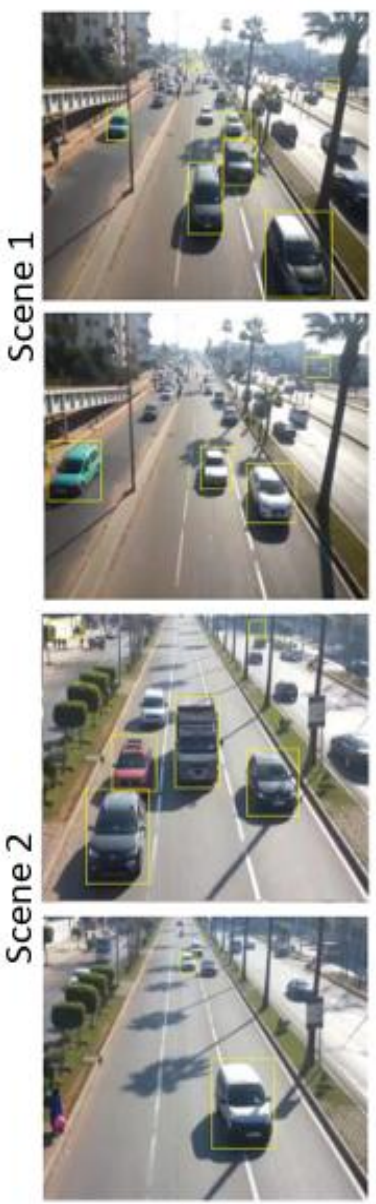

(a)
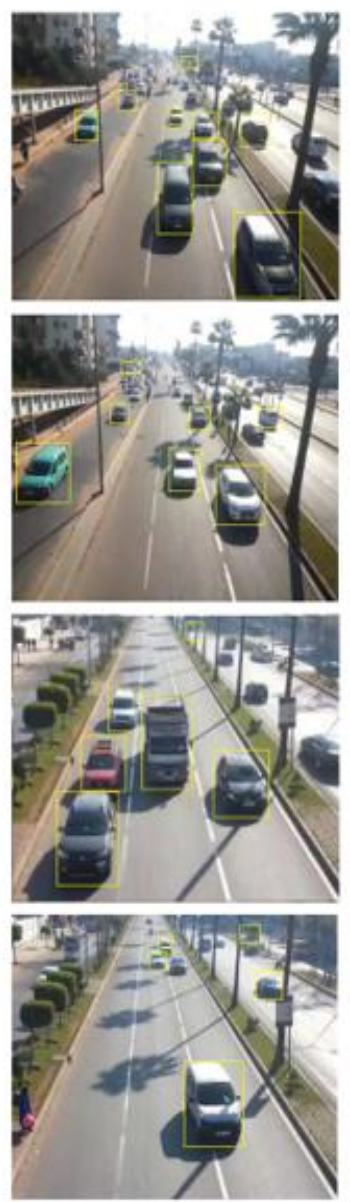

(b)
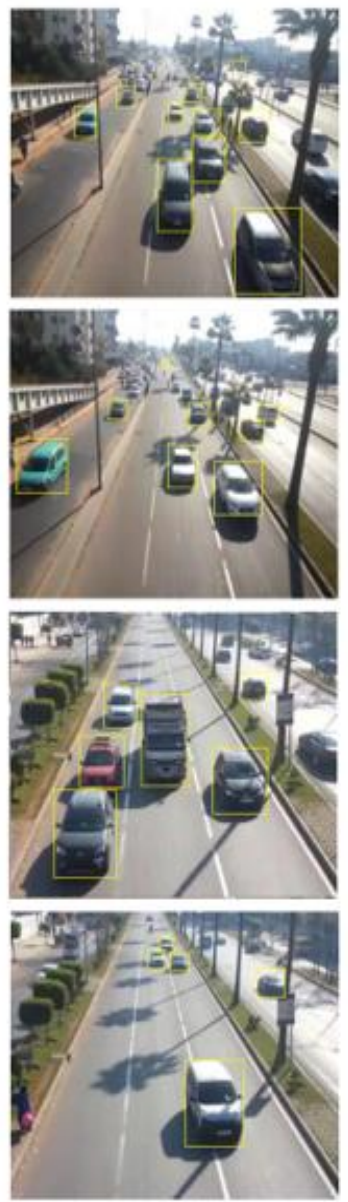

(c)
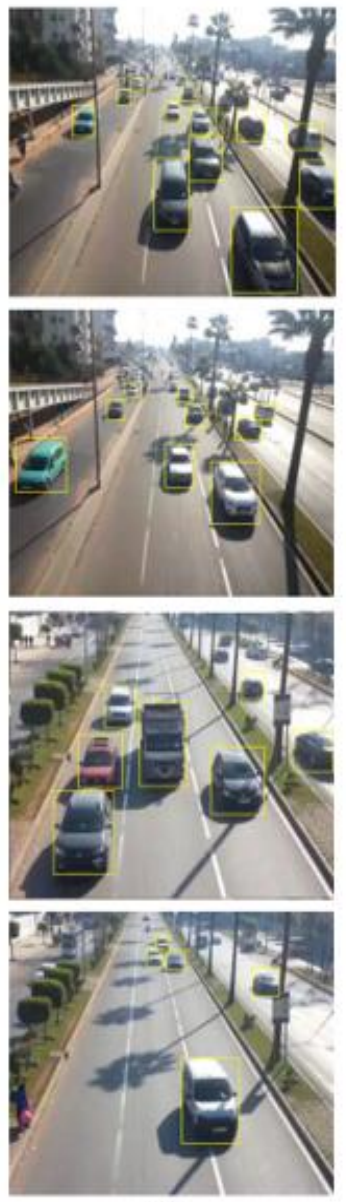

(d)

Figure 7. Vehicle detection results in different methods. (a) Faster R-CNN, (b) YOLO2, (c) SSD512, and (d) our method

As shown in Figure 7. SSD512 has better performance than Faster R-CNN and YOLO2. We also note that, our method outperforms all comparative methods. As it has high positive detection and less missing detection. To quantitatively evaluate our vehicle detection method performance, three measurements are utilized, namely Recall Rate (RR), Precision Rate (PR), and F1-score. They are defined as: 
Recall Rate $=\frac{\text { Correctly identified positive detections }}{\text { True positive detections }}$.

Precision Rate $=\frac{\text { Correctly identified positive detections }}{\text { Predicted positive detections }}$.

$\mathrm{F} 1$-score $=\frac{2 * \text { recall } * \text { precision }}{\text { recall }+ \text { precision }}$.

Table 1 shows the numerical comparison results of the four methods for vehicle detection. From Table.1, SSD512 has better precision rate than YOLO2 but it consumes more time for processing. It can be noted that our method has the best performance in all measurement parameters (RR, RP, F1-score, and time processing) because the detection of small objects is improved and the number of the network parameters are reduced.

Table 1. Accuracy comparison between different methods

\begin{tabular}{|l|l|l|l|l|l|l|l|}
\hline Methods & $\begin{array}{l}\text { Ground- } \\
\text { Truth }\end{array}$ & True Positive & $\begin{array}{l}\text { False } \\
\text { Negative }\end{array}$ & Recall Rate & $\begin{array}{l}\text { Precision } \\
\text { Rate }\end{array}$ & F1-score & $\begin{array}{l}\text { Time per } \\
\text { Frame }\end{array}$ \\
\hline Faster R-CNN & 1250 & 840 & 276 & 67.18 & 75.27 & 0.71 & 1.2 \\
\hline YOLO2 & 1250 & 905 & 235 & 72.40 & 79.38 & 0.76 & 0.4 \\
\hline SSD500 & 1250 & 948 & 193 & 75.84 & 83.08 & 0.79 & 0.49 \\
\hline Our Method & 1250 & 995 & 138 & 79.6 & 87.89 & 0.84 & 0.2 \\
\hline
\end{tabular}

\subsection{Evaluation of the vehicular queue length measurement method}

In this paper, a vehicular queue length measurement system is proposed. This system is implemented by $\mathrm{C}++$ programming language, and it runs on the $\mathrm{PC}$ equipped with core i7 processor with 8GB RAM. To evaluate our proposed system, we utilize a video which is taken from one traffic camera of Casablanca city and installed on top of the traffic light. The traffic signal cycle length is 60 second; the amount of the red time is 24 seconds. It is difficult to make a comparative study between our proposed system and previous systems, because of the different scene videos which are used in the different systems. As previously mentioned, all previous queue length measurement systems utilized traditional image processing such as edge detection and corner detection. Therefore, we will compare our system when using the vehicle detection process based on improved SSD method and when using edge detection method.

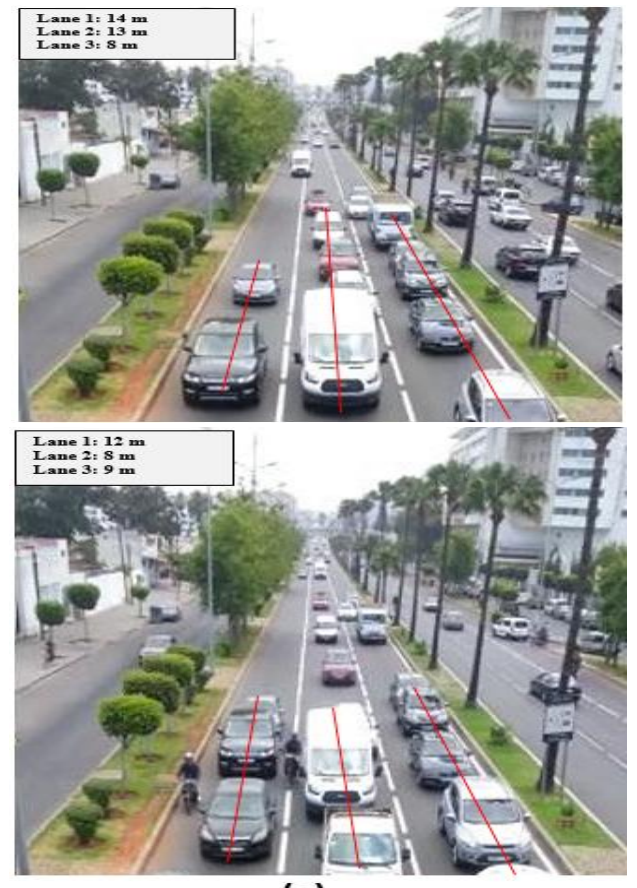

(a)
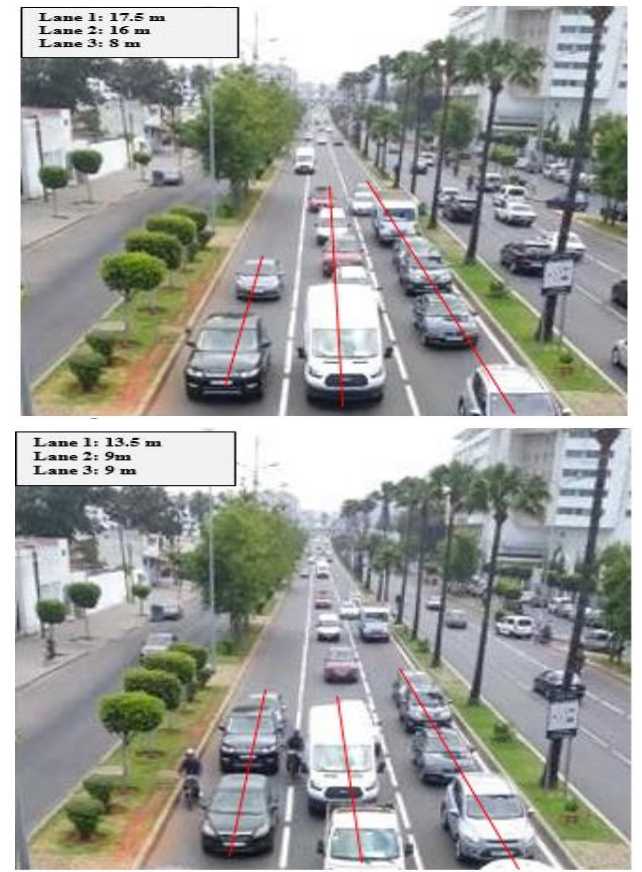

(b)

Figure 8. Examples of queue length measurement by using the two methods. (a) Queue length measurement based on edge detection method, (b) Queue length measurement based on our method 

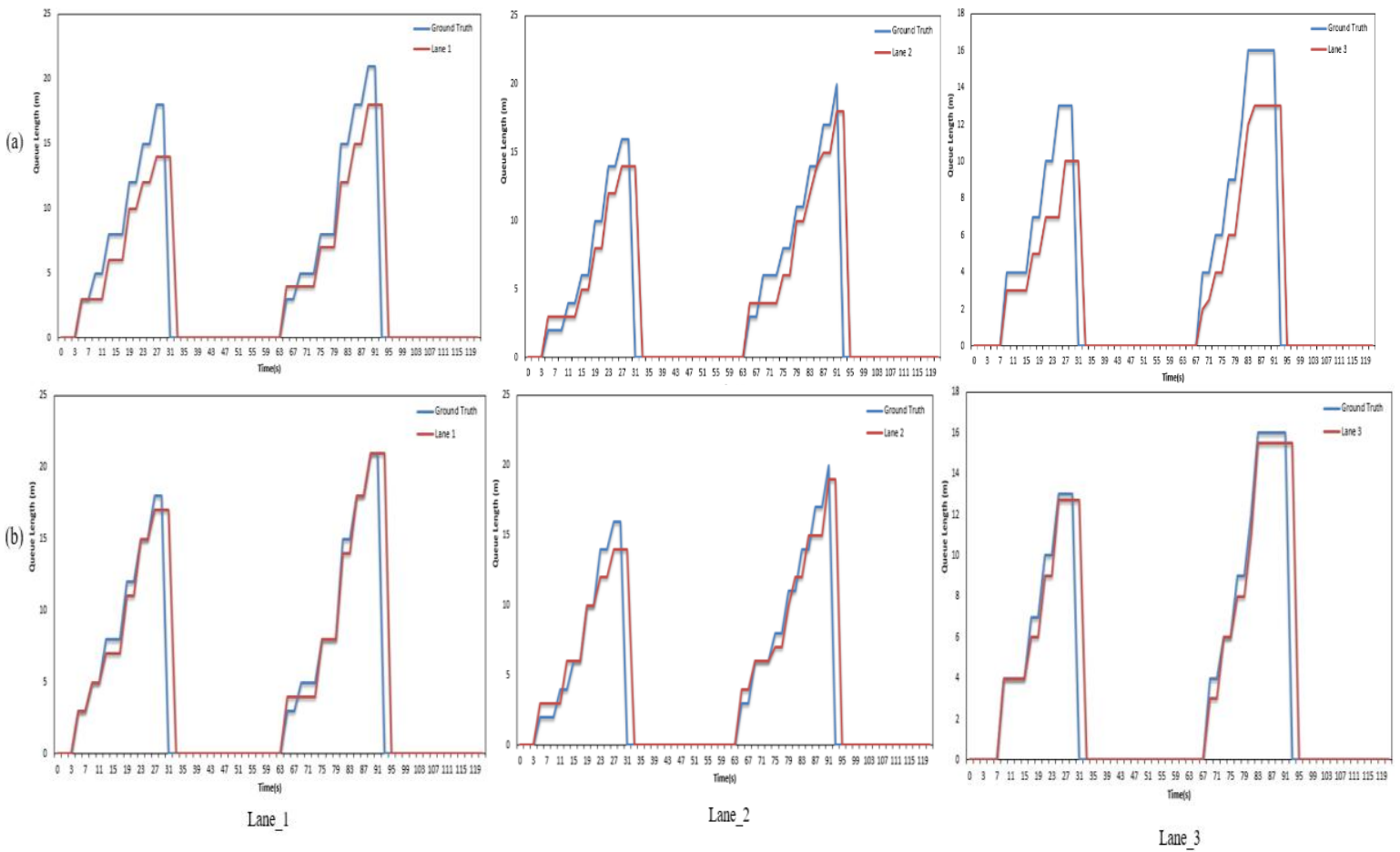

Figure 9. The results of queue length measurement of the three lanes by using the two methods for 2 traffic signals cycles.

(a) Edge detection method, (b) improved SSD method

Figure 8 shows examples of queue length measurement by using the two methods. Figure 8 (b) shows the results of applying the improved SSD method, while Figure 8 (a) shows the result of using the edge detection method. The queue length is marked by red line and the length of queue for each lane is displayed in the upper-left portion of the resulted images. From this figure, we can note that using improved SSD method has better performance than the using of edge detection method. As it measures the queue length more accurate than edge detection method.

Figure 9 shows the results of queue length measurement of the three lanes by using the two methods for 2 traffic signal cycles. Figure 9 (a) shows the results of using the edge detection method (The result of edge detection is marked by red, while the ground-truth queue length is marked by blue), while Figure 9 (b) shows the results of applying the improved SSD method (The result of SSD is marked by red, while the ground-truth queue length is marked by blue). We notice that the proposed system starts to detect the queue when there is no motion in the head of the queue and the vehicles are detected when the traffic light is red. Then the queue length increases until the motion is detected in the head of the queue when the traffic light turns green, the queue length falls to zero with a small delay to improve the stability of the system as previously mentioned. As noted from the figure, the queue length measurement by using SSD method precisely follows the ground-truth queue length better than the edge detection method. The experimental results manifest that the proposed system has the ability for measuring the queue length accurately and reliability in real time.

\section{Conclusion}

In this paper, an image processing in real-time system has been proposed to detect and measure the vehicular queue length. The proposed system consists of two main steps: motion detection, and vehicle detection. In the first step, we utilized frame differencing algorithm, while in the step two, we detected the vehicles by using SSD network after applying some modifications on it to fit with in our system and to improve the accuracy of vehicle detection process. After applying our vehicle detection method, we noted that the accuracy of our system increased, as it has high positive detection and less missing detection. The experiments demonstrate that this system is able to accurately detect and measure the vehicular queue length. 


\section{References}

1. Burnos, P., Gajda, J., Piwowar, P., Sroka, R., Stencel, M., Zeglen, T. (2007) Measurements of road traffic parameters using inductive loops and piezoelectric sensors.

2. Sun, C., Ritchie, S. G. and Tsai, K. (1998) Algorithm development for derivation of section-related measures of traffic system performance using inductive loop detectors. Transportation Research Record, 1643(1), pp. 171-180.

3. Slimani, I., Zaarane, A., Hamdoun, A., Atouf, I. (2018) Traffic surveillance system for vehicle detection using discrete wavelet transform. Journal of Theoretical \& Applied Information Technology, 96(17).

4. AL Okaishi, W., Zaarane, A., Slimani, I., Atouf, I., Benrabh, M. (2019a) Vehicular queue length measurement based on edge detection and vehicle feature extraction. Journal of Theoretical \& Applied Information Technology, 97(5).

5. Fathy, M., Siyal, MY. (1995) Real-time image processing approach to measure traffic queue parameters. IEE Proceedings-Vision, Image and Signal Processing, 142(5), pp. 297-303.

6. Albiol, A., Albiol, A., Mossi, J. M. (2011a) Video-based traffic queue length estimation. Computer Vision Workshops (ICCV Workshops), 2011 IEEE International Conference on. IEEE, pp. 1928-1932.

7. Chintalacheruvu, N., Muthukumar, V. (2012) Video based vehicle detection and its application in intelligent transportation systems. Journal of Transportation Technologies, 2(04), p. 305.

8. Albiol, A., Sanchis, L., Albiol, A., Mossi, J. M. (2011b) Detection of parked vehicles using spatiotemporal maps. IEEE Transactions on Intelligent Transportation Systems, 12(4), pp. 1277-1291.

9. Rourke, A., Bell, M. (1991) Queue detection and congestion monitoring using image processing. Traffic engineering \& control, 32(9).

10. Siyal, M. Y., Fathy, M. (1999) A neural-vision based approach to measure traffic queue parameters in real-time. Pattern Recognition Letters, 20(8), pp. 761-770.

11. Iwasaki, Y. (1997) An image processing system to measure vehicular queues and an adaptive traffic signal control by using the information of the queues. Intelligent Transportation System, ITSC'97., IEEE Conference on. IEEE, pp. 195-200.

12. Zanin, M., Messelodi, S., Modena, C. M. (2003) An efficient vehicle queue detection system based on image processing. null. IEEE, p. 232.

13. Shirazi, M. S., Morris, B. (2015) Vision-based vehicle queue analysis at junctions. Advanced Video and Signal Based Surveillance (AVSS), 2015 12th IEEE International Conference on. IEEE, pp. 1-6.

14. Sen-Ching, S. C., Kamath, C. (2004) Robust techniques for background subtraction in urban traffic video. Visual Communications and Image Processing. International Society for Optics and Photonics, 5308, pp. 881-893.

15. AL Okaishi, W., Atouf, I., Benrabh, M. (2019b) Real-Time Traffic Light Control System Based on Background Updating and Edge Detection. 2019 International Conference on Wireless Technologies, Embedded and Intelligent Systems (WITS) on. IEEE, pp. 1-5.

16. Lowe, D. (2004) Distinctive image features from scale-invariant keypoints. International journal of computer vision, 60, pp. 91-110.

17. Dalal, N., Triggs, B. (2005) Histograms of oriented gradients for human detection. IEEE computer society conference on computer vision and pattern recognition (CVPR'05), 1, pp. 886-893.

18. Tan, X., Triggs, B. (2007) Fusing Gabor and LBP feature sets for kernel-based face recognition. International workshop on analysis and modeling of faces and gestures, pp. 235-249.

19. Nogueira, K., Miranda, W. O., Dos Santos, J. A. (2015) Improving spatial feature representation from aerial scenes by using convolutional networks. Graphics, Patterns and Images (SIBGRAPI), $201528^{\text {th }}$ SIBGRAPI Conference on, IEEE, pp. 289-296.

20. Krizhevsky, A., Sutskever, I., Hinton, G. E. (2012) Imagenet classification with deep convolutional neural networks. Neural Information Processing Systems, pp. 1106-1114.

21. Simonyan, K., Zisserman, A. (2014) Very deep convolutional networks for large-scale image recognition," arXiv preprint arXiv: 1409.1556.

22. Girshick, R., Donahue, J., Darrell, T. (2015a) Region-based convolutional networks for accurate object detection and segmentation. IEEE transactions on pattern analysis and machine intelligence, 38(1), pp. 142-158.

23. Girshick, R. (2015b) Fast R-CNN. IEEE International Conference on Computer Vision (ICCV), pp. 1440-1448.

24. Ren, S., HE, K., Girshick, R., Sun, J. (2015) Faster R-CCN: Towards real-time object detection with region proposal networks. Advances in neural information processing systems, pp. 91-99. 